\title{
MONITOREO DEL SUEÑO EN CONDUCTORES DE ÓMNIBUS Y CAMIONES: FACTOR RELEVANTE A CONSIDERAR PARA LA RENOVACIÓN DE LA LICENCIA DE CONDUCIR
}

\author{
Jorge Rey de Castro ${ }^{1,2,3, a, b}$, Edmundo Rosales-Mayor ${ }^{1,2,3, b, c}$
}

\begin{abstract}
RESUMEN
La información publicada indica que hasta un tercio de los accidentes de tránsito son producidos por la somnolencia del conductor. El síndrome de apnea-hipopnea del sueño (SAHS) es una enfermedad orgánica que causa somnolencia. Puede diagnosticarse por medio de un registro del sueño y controlarse con modalidades terapéuticas distintas y de diferente complejidad según su gravedad, lo que determina el costo final del manejo del SAHS. En los pacientes que emplean disciplinadamente la terapia, los resultados son muy buenos y logran controlar la somnolencia, mejoran la calidad de vida del afectado, protegen su salud y disminuyen ostensiblemente el riesgo de accidentes durante la conducción debido a somnolencia. Ponemos en consideración de las autoridades responsables la aplicación de estas pruebas en choferes con sospecha de SAHS que renuevan licencias de conducir de tipo A-II y A-III.
\end{abstract}

Palabras clave: Transtornos por excesiva somnolencia; Accidentes de tránsito; Apnea del sueño obstructiva; Polisomnografía; Perú (fuente: DeCS BIREME).

\section{SLEEP MONITORING IN BUS AND TRUCK DRIVERS: RELEVANT FACTOR TO CONSIDER FOR THE RENEWAL OF THE DRIVING LICENSE}

\begin{abstract}
The available information indicates that up to one third of the road traffic accidents are produced by the driver's sleepiness. Sleep apnea-hypopnea syndrome (SAHS) is an organic disease that causes sleepiness. It can be diagnosed by a sleep register and can be controlled by different therapeutic means, varying their complexity according to its severity, which determines the final cost of the management of SAHS. In patients using the therapy thoroughly, the results are very good and achieve to control the sleepiness, improve the quality of life of the affected subject, protect his health and markedly decrease the risk of accidents due to sleepiness during driving. We put into consideration of the respective authorities the application of these tests in drivers suspected of having SAHS who renew their driving licenses type A-II and A-III.
\end{abstract}

Key words: Disorders of excessive somnolence; Accidents, traffic; Sleep apnea, obstructive; Polysomnography; Peru (source: MeSH NLM).

\section{INTRODUCCIÓN}

Según el Informe Mundial sobre prevención de los traumatismos causados por el tránsito, se estima que 1,2 millones de personas mueren y hasta 50 millones resultan heridas debido a accidentes de tránsito ocurridos en la vía pública cada año. Esta misma fuente menciona que de no implementarse medidas de carácter preventivo las cifras mencionadas aumentarían un $65 \%$ en los próximos 20 años ${ }^{(1)}$.

El cansancio y la somnolencia de los conductores durante la conducción han sido descritas como causa de accidentes de tránsito en la ciudad y las carreteras, estando debidamente sustentada en publicaciones internacionales (2-5). Un conductor somnoliento disminuye progresivamente su capacidad de atención y concentración durante el manejo y pierde capacidad de respuesta ante condiciones específicas que exigen reacciones inmediatas cuando circula por la ciudad o la carretera. El pestañear y dormitar durante la conducción expresan un nivel extremo de deuda de sueño ${ }^{(6)}$, usualmente, los accidentes producidos en estas circunstancias tienen alta siniestralidad en términos de pasajeros muertos, heridos y pérdidas materiales. La información publicada en el exterior indica que de 4 a

\footnotetext{
Centro de Trastornos Respiratorios del Sueño (CENTRES), Clínica Anglo Americana. Lima, Perú.

Facultad de Medicina, Universidad Peruana Cayetano Heredia. Lima, Perú.

3 Grupo de Investigación en Sueño (GIS). Lima, Perú.

a Médico neumólogo; ${ }^{b}$ Magister en Medicina; ${ }^{c}$ Médico, Magister en Sueño.
} 
$30 \%$ de los accidentes en carreteras son producidos por la somnolencia durante la conducción de vehículos ${ }^{(7)}$.

Las causas más frecuentes de accidentes debidos a somnolencia del conductor son privación aguda del sueño, hábitos del conductor durante su trabajo como, manejar durante la noche, en horario vespertino o jornadas prolongadas sin el descanso requerido, uso de medicación como sedantes o hipnóticos o antidepresivos tricíclicos y antihistamínicos, consumo de alcohol, desórdenes orgánicos del sueño no tratados o no identificados como el síndrome de apnea-hipopnea del sueño (SAHS) o la narcolepsia ${ }^{(8-10)}$.

Las características que rodean al escenario son: el accidente ocurre generalmente entre las 00.00-7.00 horas y en horario vespertino entre las 13:00-15:00 horas, el conductor maneja solo, la unidad sale inadvertidamente de la ruta o invade el carril contrario y, por último, el conductor no deja huellas de haber intentado alguna maniobra evasiva para evitar la colisión (11-14). Dado que el conductor cansado o somnoliento no tiene capacidad de hacer maniobras evasivas para evitar la colisión, estos accidentes están asociados con una alta morbilidad, mortalidad y elevados costos debido a la destrucción de las unidades.

Según la Dirección General de Circulación Terrestre del Ministerio de Transportes y Comunicaciones (MTC) de Perú, en el año 2007 se produjeron 3510 muertes y 49857 heridos a consecuencia de accidentes de tránsito ${ }^{(15)}$. Esta misma fuente señala que el año 2008 se registraron 85337 accidentes en el país con un incremento del $6,7 \%$ con respecto al año anterior. Tanto el número de heridos y muertos causados por los accidentes de tránsito a lo largo de los últimos treinta y cinco años tiene una clara tendencia a incrementarse cada año, a un factor de 3,59 veces por año ${ }^{(16)}$. El Informe Defensorial 108 documenta claramente la alta siniestralidad ocasionada por los accidentes de ómnibus interprovinciales comparados con el total de accidentes registrados en la red nacional vial entre los años 2002-2005. Un accidente provocado por un ómnibus interprovincial causa 2,63 veces más heridos y 2,33 más muertos que el resto de unidades ${ }^{(17)}$.

\section{LA SOMNOLENCIA Y LOS ACCIDENTES DE TRÁNSITO EN CARRETERAS DEL PERÚ}

La información obtenida en los últimos años indica que la somnolencia durante la conducción sería causa importante de accidentes de tránsito en las carreteras de nuestro país ${ }^{(18-24)}$. Esto indica las causas, aunque no es posible precisar la cifra exacta de esta causa en relación con las ya conocidas. En este escenario se han hecho propuestas concretas a las autoridades competentes del Ministerio de Salud para corregirlo y de esa manera disminuir el riesgo por accidentes debido a somnolencia o cansancio ${ }^{(24)}$.

Como ha sido señalado por la literatura foránea, una enfermedad orgánica como el SAHS, es factor de riesgo de accidentes debido a la somnolencia que esta condición clínica produce ${ }^{(25-27)}$. No tenemos certeza de las prevalencias de esta enfermedad en población general o en conductores de nuestro medio dado que no se han implementado estudios con este objetivo. Sin embargo, tomando como base la frecuencia de los síntomas cardinales de esta enfermedad, como frecuencias del ronquido, pausas respiratorias durante el sueño y somnolencia diurna registradas por cuestionario en población adulta supuestamente sana, parece indicar que esta enfermedad tendría una alta frecuencia ${ }^{28)}$. En el estudio de Liendo y Castro, la frecuencia de ronquido y pausas respiratorias (por cuestionario) encontradas en conductores formales e informales de ómnibus de Lima fue $55-58 \%$ y $24-25 \%$, respectivamente ${ }^{(23)}$.

La capacidad de predicción de enfermedad a partir de la sintomatología, aplicación de cuestionarios, examen físico y uso de ecuaciones predictivas tienen limitaciones. A pesar de ello, es necesario proponer pautas generales que contribuyan a la identificación y ulterior tratamiento de esta condición médica. Las propuestas elaboradas en este manuscrito podrían ser empleadas por el personal médico responsable que evalúa postulantes para renovar la licencia de conducir A-II y A-III.

\section{PROPUESTA}

Los conductores que renuevan licencias de conducir A-II y A-III ${ }^{29)}$, requieren la evaluación médica obligatoria pautada por el MTC. En esta circunstancia el médico responsable podría aplicar los criterios aquí planteados con la finalidad de identificar conductores con sospecha de SAHS que requieran evaluación por especialista en trastornos del sueño. El estudio requerido puede ser la polisomnografía (PSG) o poligrafía respiratoria $(P R)^{(30-34)}$. Estas pruebas permiten registrar variables neurofisiológicas y cardiorrespiratorias en el primer caso y exclusivamente cardiorrespiratorias en el segundo. La lectura y análisis del registro realizado durante toda la noche permite identificar de manera objetiva a conductores que tienen SAHS y su respectiva gravedad.

\section{LA POLISOMNOGRAFÍA}

Esta es la prueba de sueño operativamente más compleja y requiere que el paciente obligatoriamente 
duerma en una habitación acondicionada con ese fin. El estudio es supervisado por una enfermera especialista a lo largo de toda la noche. Si bien es cierto esta es la prueba estándar aceptada internacionalmente tiene muy alto costo, lo que limita su empleo en sectores de la población que no pueden sufragar los elevados gastos.

El arreglo polisomnográfico debe incluir por lo menos los siguientes canales de registro: C4A1, C3A2, O4A1, O3A2, dos canales de electrooculograma, electromiografía del mentón, electromiografía de músculos tibiales anteriores derecho e izquierdo, flujo oronasal con thermistor y cánula de presión nasal, ronquido, esfuerzo torácico y abdominal con bandas piezoeléctricas, electrocardiograma en derivación II, $\mathrm{SatO}_{2} \mathrm{Hb}$ por medio de pulsioximetría de la hemoglobina con tiempo de muestreo de cuatro segundos y posición corporal. El estudio debe implementarse bajo supervisión de personal especializado en esta prueba. De acuerdo a estas características el estudio la polisomnografía cumple con los criterios de prueba tipo I según clasificación de la American Sleep Disorders Association (ASDA) ${ }^{(35)}$.

La clasificación de los estadios del sueño se realiza en forma manual utilizando los criterios de Rechtschaffen y Kales (36) o los de la Academia Americana de Medicina

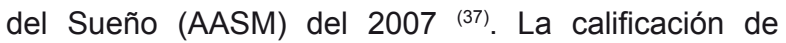
despertares deberán sustentarse en las guías de la ASDA (38). Una vez finalizado el registro, todas las pruebas deben ser revisadas y calificadas de forma manual por el médico especialista responsable.

El informe de la PSG debe incluir la fecha del estudio con la cuantificación de los siguientes parámetros: tiempo total de registro, tiempo total de sueño, tiempo total de vigilia intrasueño, latencia del sueño, latencia REM e índice de despertares EEG, tiempo en minutos y porcentajes de vigilia, sueño no REM (fase 1,2,3,4) y sueño REM si se emplea el sistema de estadiaje de Rechtschaffen y Kales (36) o vigilia, N1, N2, N3 y REM si se usa el método propuesto por la AASM (37). Índice de apnea hipopnea (IAH) durante el sueño, sueño noREM, sueño REM, decúbito supino y decúbito lateral. El índice de disturbio respiratorio que incluye el valor promedio por hora de la suma de apneas, hipopneas y los esfuerzos respiratorios asociados a despertares. La desaturación máxima durante el sueño y porcentaje del tiempo total de sueño con saturación por debajo de $90 \%$. Finalmente los movimientos periódicos de miembros asociados a despertares EEG. El tiempo total de sueño no debe ser menor de seis horas.

El informe final debe estar firmado y sellado por el médico especialista en trastornos del sueño. El laboratorio de sueño debe guardar en formato informático, todas y cada una de las copias del estudio para una eventual auditoria por el MTC o Ministerio de Salud (MINSA).

\section{LA POLIGRAFÍA RESPIRATORIA}

Los estudios de PR son dispositivos de nivel III (35) según la clasificación de la ASDA. Estos equipos tienen la ventaja de registrar el monitoreo del sueño en ambientes hospitalarios convencionales o en el domicilio del conductor y sin supervisión de personal especializado. Este tipo de dispositivo tiene un costo mucho más bajo que la polisomnografía lo que la hace operativamente manejable en poblaciones más grandes.

El equipo a emplear debe tener como mínimo los siguientes canales: flujo nasal por medio de thermistor o cánula nasal, ronquido, esfuerzo toráxico, posición y oximetría de pulso. Una vez finalizado el registro, todas las pruebas deben ser revisadas y calificadas de forma manual por el médico especialista responsable. Es aconsejable que el equipo este validado con la PSG.

El informe final debe incluir la fecha del estudio, cuantificación del número de apneas e hipopneas obstructivas, apneas mixtas y centrales, tiempo total de registro, tiempo de registro con SatO2 $\mathrm{Hb}$ menor de $90 \%$, SatO2 $\mathrm{Hb}$ media, índice de apneas, IAH, desaturación máxima durante el sueño. El tiempo total de registro no debe ser menor de seis horas.

De la misma forma que la PSG, el informe final estará firmado y sellado por el médico especialista en trastornos del sueño, el laboratorio de sueño deberá guardar en formato informático, todas y cada una de las copias del estudio para una eventual auditoria por el MTC o MINSA.

Ambas pruebas, la PSG y la $\mathrm{PR}$, sirven para determinar presencia o ausencia de enfermedad como ha sido demostrado en la literatura médica ${ }^{(33,39-42)}$.

\section{INDICACIONESPARAESTUDIODEMONITOREO DEL SUEÑO}

Las variables a considerar son: índice de masa corporal, valor de la presión arterial, configuración anatómica de la faringe de acuerdo con la clasificación de Mallampati (43-44), circunferencia del cuello, puntaje de la escala de somnolencia Epworth la cual ha sido validada en el Perú ${ }^{(45)}$ y presencia de ronquido durante el sueño.

- Índice de Masa Corporal $\geq 30$, calculado de la fórmula: (peso en $\mathrm{kg}$ )/(talla en $\mathrm{cm})^{2}$. 

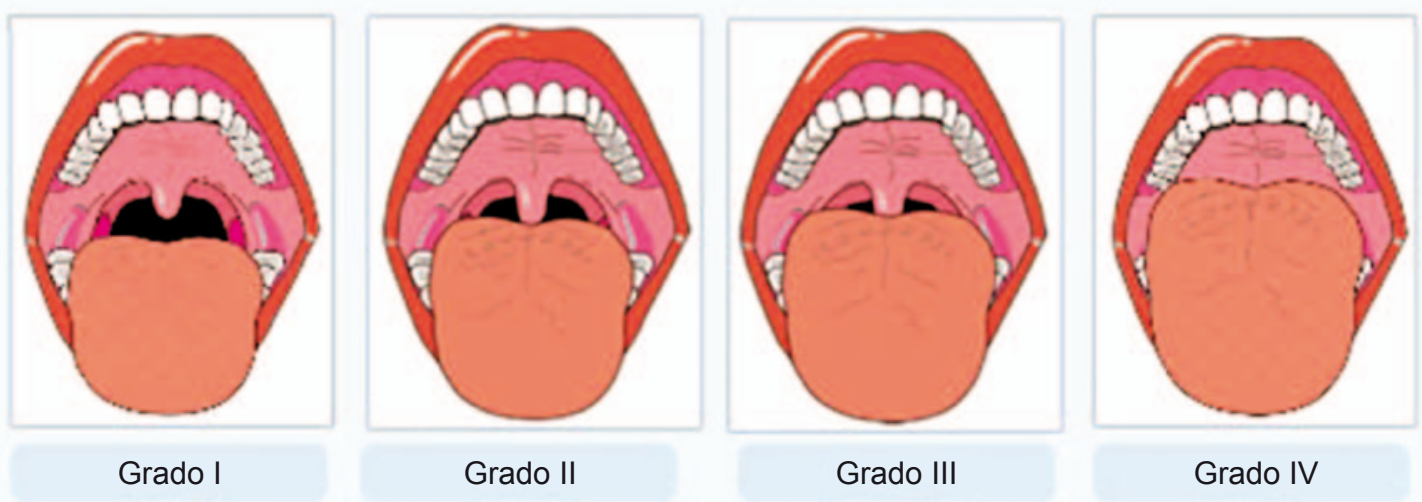

\begin{tabular}{cll} 
Clase & Visualización directa, paciente sentado. & Imagen laringoscópica. \\
\hline I & Paladar blando, fauces, úvula, pilares. & Toda la glotis. \\
II & Paladar blando, fauces, úvula. & Comisura posterior. \\
III & Paladar blando y base de úvula. & Punta de epiglotis. \\
IV & Solo paladar duro. & No se observa estructura glótica. \\
\hline
\end{tabular}

Figura 1. Clasificación de Mallampati.

Tomado de: Mallampati SR, Gatt SP, Gugino LD, Desai SP, Waraksa B, Freiberger D, et al. A clinical sign to predict difficult tracheal intubation: a prospective study. Can Anaesth Soc J. 1985; 32(4):429-34.

- Presión arterial sistólica (PAS) $\geq 140 \mathrm{mmHg}$ o presión arterial diastólica (PAD) $\geq 90 \mathrm{mmHg}$ de acuerdo con los criterios del séptimo reporte de de la JNC ${ }^{(46)}$.

- Orofaringe con clasificación de Mallampati III o IV (43-44) (Figura 1).

- Perímetro del cuello en centímetros > $45 \mathrm{~cm}$ en hombres y $>38 \mathrm{~cm}$ en mujeres. La medición debe hacerse con una cinta métrica y a la altura del cartílago cricoides.

- Escala de somnolencia Epworth (versión peruana modificada) ${ }^{(45)}$ con puntaje mayor o igual a 12 (Figura 2)

- Ronquido intenso atestiguado por la pareja o familiares en por lo menos cinco noches por semana.

El conductor que postula para la renovación de las licencias de conducir y que cumpliera tres o más de los criterios señalados debe ser evaluado por el especialista en trastornos del sueño para la implementación de una de las pruebas arriba descritas.

EI SAHS puede controlarse con el tratamiento que por cierto es implementado de acuerdo con la gravedad de la enfermedad. En líneas generales, el tratamiento incluye bajar de peso, no beber alcohol dos horas antes de dormir así sea en pequeñas cantidades, dejar de fumar, no emplear medicación para dormir como hipnóticos, sedantes o tranquilizantes. Las formas leves de la enfermedad pueden ser tratadas con dispositivos de avance mandibular. Los casos con formas moderadas o graves de enfermedad requieren usar dispositivos de presión positiva sobre la vía aérea, llamados CPAP. Está demostrado que el tratamiento del SAHS mejora la calidad del sueño, calidad de vida y protege al paciente del riesgo de accidentes de tránsito durante la conducción ${ }^{(47-49)}$. Los documentos de consenso de instituciones académicas y estatales de prestigio no dudan de la eficacia de esta modalidad de tratamiento y su impacto en la reducción del riesgo de accidentes es pacientes con SAHS ${ }^{(50-52)}$.

\section{VIABILIDAD DE LA PROPUESTA}

Stephen et al. en una revisión sistemática de la literatura y metaanálisis han determinado variables predictivas de accidentes de tránsito en choferes afectados por el SAHS. De acuerdo con esta publicación las variables son: índice de masa corporal, índice de apnea hipopnea (número de apneas e hipopneas por hora de sueño), desaturación de oxígeno y somnolencia ${ }^{(53)}$. Sin embargo, no es posible realizar una prueba de monitoreo del sueño a todos los conductores por lo que se propone emplear variables clínicas que permitan distinguir formas de enfermedad que pongan en riesgo a los conductores y pasajeros. De acuerdo con ello el grupo de De Mello en Brasil ha recomendado utilizar en los exámenes médicos de los conductores, variables subjetivas y objetivas que permitan seleccionar a choferes con alto riesgo de presentar SAHS ${ }^{(54)}$. 


\section{Escala de Somnolencia Epworth \\ (Versión Peruana Modificada)}

¿Qué tan probable es que usted cabecee o se quede dormido en las siguientes situaciones? Considere los últimos meses de sus actividades habituales. No se refiere a sentirse cansado debido a actividad física. Aunque no haya realizado últimamente las situaciones descritas, considere como le habrían afectado. Use la siguiente escala y marque con una X la opción más apropiada para cada situación:

- Nunca cabecearía

- Poca probabilidad de cabecear

- Moderada probabilidad de cabecear

- Alta probabilidad de cabecear

\begin{tabular}{|c|c|c|c|c|}
\hline \multirow{2}{*}{ Situación } & \multicolumn{4}{|c|}{ Probabilidad de cabecear } \\
\hline & Nunca & Poca & Moderada & Alta \\
\hline \multicolumn{5}{|l|}{ Sentado leyendo } \\
\hline \multicolumn{5}{|l|}{ Viendo televisión } \\
\hline \multicolumn{5}{|c|}{$\begin{array}{l}\text { Sentado (por ejemplo en el teatro, enuna reunión, en el cine, en una } \\
\text { conferencia, escuchando la misa o el culto) }\end{array}$} \\
\hline \multicolumn{5}{|c|}{$\begin{array}{l}\text { Como pasajero en un automóvil, ómnibus, micro o combi durante una hora } \\
\text { o menos de recorrido }\end{array}$} \\
\hline \multicolumn{5}{|c|}{ Recostado en la tarde si las circunstancias lo permiten } \\
\hline \multicolumn{5}{|l|}{ Sentado conversando con alguien } \\
\hline \multicolumn{5}{|l|}{ Sentado luego del almuerzo y sin haber bebido alcohol } \\
\hline \multicolumn{5}{|c|}{$\begin{array}{l}\text { Conduciendo el automóvil cuando se deitene algunos minutos por razones } \\
\text { de tráfico }\end{array}$} \\
\hline Parado y apoyándose o no en una pared o mueble & & & & \\
\hline
\end{tabular}

¿Usted maneja vehículos motorizados (auto, camioneta, ómnibus, micro, combi, etc.)?

$$
\text { ( )sí ( ) NO }
$$

\section{Gracias por su cooperación}

Validación Peruana de la Escala de Somnolencia de Epworth. Rosales E. Rey de Castro J. Huayanay L. y Zagaceta K. Octubre 2009.

Figura 2. Escala de Somnolencia Epworth -versión peruana modificada ${ }^{(45)}$.

NOTA: Cada situación tiene un puntaje de 0 a 3 . Cero indica nunca y 3 una alta probabilidad de cabecear. El puntaje total se obtiene sumando el puntaje de cada situación. Para personas que manejan vehículos motorizados, se suma los primeros ocho ítems. Para personas que no manejan vehículos motorizados, se suma los primeros siete ítems y el ítem nueve.

Las recomendaciones no han sido validadas lo que constituye una limitación. Sin embargo, es necesario que los médicos responsables dispongan de información y herramientas que les permitan sospechar la presencia de esta enfermedad. Esta propuesta tiene en el Perú limitaciones operativas concretas que es necesario subrayar. A la fecha en que se redacta este artículo sólo cinco centros especializados en sueño implementan los dos tipos de prueba mencionadas, cuatro médicos neumólogos hacen exclusivamente poligrafías respiratorias en Lima Metropolitana y hay sólo un especialista en Piura. En el ámbito MINSA sólo existe un centro especializado y ninguno en EsSalud ni centros de las Fuerzas Armadas. La demanda que supondría la aplicación de esta sistemática de identificación de enfermedad no podría ser cubierta por las razones antes mencionadas y es, en tal sentido, muy importante que los principales hospitales del MINSA, EsSalud y Fuerzas Armadas pongan operativos sus propios centros especializados en sueño que no se limitarían exclusivamente a identificar SAHS en choferes sino que también permitirian establecer un sinnúmero de enfermedades asociadas al sueño en el área asistencial.

De otro lado, los costos del estudio deben ser cubiertos por las empresas de transportes o por el seguro social, si el conductor tiene la prestación social referida en el marco de la formalización del transporte terrestre. Sólo identificando la enfermedad y tratándola se podrá controlar el riesgo. Es indispensable que los propietarios asuman dicha responsabilidad para el 
caso de trabajadores que no tienen seguro formal y que contratan temporalmente, como garantía de seguridad para sus pasajeros.

\section{AGRADECIMIENTOS}

Agradecemos al Dr. J. Jaime Miranda por la revisión y recomendaciones al manuscrito.

\section{Fuente de Financiamiento \\ Autofinanciada.}

\section{Conflicto de Interés}

Jorge Rey de Castro es director y propietario de CENTRES (Centro de Trastornos Respiratorios del Sueño) de la Clínica Anglo Americana en Lima - Perú.

\section{REFERENCIAS BIBLIOGRÁFICAS}

1. Peden MM, Scurfield R, Sleet D, Mohan D, Hyder AA, Jarawan E, Mathers C. World report on road traffic injury prevention. Geneva: World Health Organization; 2004.

2. Lyznicki JM, Doege TC, Davis RM, Williams MA. Sleepiness, driving, and motor vehicle crashes. Council on Scientific Affairs, American Medical Association. JAMA. 1998; 279(23): 1908-13.

3. Dement WC, Mitler MM. It's time to wake up to the importance of sleep disorders. JAMA. 1993; 269(12): 1548-50.

4. Sagberg F. Road accidents caused by drivers falling asleep. Accid Anal Prev. 1999; 31(6): 639-49.

5. Horne JA, Reyner LA. Sleep related vehicle accidents. BMJ. 1995; 310(6979): 565-67.

6. Brown ID. Driver fatigue. Hum Factors. 1994; 36(2): 298314.

7. Mitler MM, Miller JC, Lipsitz JJ, Walsh JK, Wylie CD. The sleep of long-haul truck drivers. N Engl J Med. 1997; 337(11): 755-61.

8. Lertzman M, Wali SO, Kryger M. Sleep apnea a risk factor for poor driving. CMAJ. 1995; 153(8): 1063.

9. American Thoracic Society. Sleep apnea, sleepiness and driving risk. Am J Respir Crit Care Med. 1992; 150: 1463-73.

10. Findley LJ, Unverzagt ME, Suratt PM. Automobile accidents involving patients with obstructive sleep apnea. Am Rev Respir Dis. 1988; 138(2): 337-40.

11. National Sleep Foundation. Drowsy driving: detection and prevention. [Página Web] Washington DC: NSF; 2010. [Actualizado 2010; Fecha de acceso: 01 de abril del 2010]; Disponible en: http://drowsydriving.org/about/detectionand-prevention/.

12. McCartt AT, Rohrbaugh JW, Hammer MC, Fuller SZ. Factors associated with falling asleep at the wheel among long-distance truck drivers. Accid Anal Prev. 2000; 32(4): 493-504.

13. Johnson EO. Sleep in America: 1999. Results from the Nacional Sleep Foundations 1999 Omnibus Sleep Poll. Washington DC: National Sleep Foundation; 1999.

14. National Sleep Foundation. 2002 "Sleep in America" Poll: Adult Sleep Habits. Washington DC: National Sleep Foundation; 2002.
15. Perú, Ministerio de Transportes y Comunicaciones. Perú: Número de accidentes de tránsito fatales y no fatales por año según causa 2000-2008. Lima, Ministerio de Transportes y Telecomunicaciones; 2009.

16. Miranda JJ, Huicho L, Lopez L, Paca A, Luna D, Rosales $\mathbf{E}$, et al. Incidencia, tendencia de los accidentes de tránsito en el Perú y factores de riesgo dependientes del peatón, vehículo y conductor [Informe Técnico]. Lima: Instituto Nacional de Salud, Salud Sin Límites Perú; 2009.

17. Defensoría del Pueblo. Informe Defensorial $N^{\circ} 108$ - Pasajeros en riesgo: La seguridad en el transporte interprovincial. Lima: Defensoría del Pueblo; 2008.

18. Rey de Castro J. Accidentes de tránsito en carreteras e hipersomnia durante la conducción. ¿Es frecuente en nuestro medio? La evidencia periodística. Rev Med Hered. 2003;14(2):69-73.

19. Rey de Castro J, Soriano S. Hipersomnia durante la conducción de vehículos ¿causa de accidentes en carreteras? A propósito de un estudio cualitativo. Rev Soc Peru Med Interna. 2002;15(3):142-9.

20. Soriano S, Rey de Castro J. Una aproximación a los choferes de omnibuses interprovinciales. Accidentes de tránsito y privación crónica del sueño en la carretera Panamericana. Antropológica. 2002; 20(1): 231-46.

21. Rey de Castro J, Gallo J, Loureiro H. Cansancio y somnolencia en conductores de ómnibus y accidentes de carretera en el Perú: estudio cuantitativo. Rev Panam Salud Publica. 2004;16(1):11-8.

22. Rosales E, Egoavil M, Durand I, Montes N, Flores R, Rivera S, et al. Accidentes de carretera y su relación con cansancio y somnolencia en conductores de ómnibus. Rev Med Hered. 2009;20(2):136-47.

23. Liendo G, Castro C. Cansancio y somnolencia en conductores de ómnibus interprovinciales con base en Lima, según condiciones laborales de la empresa de transportes [Tesis de Bachiller]: Universidad Peruana Cayetano Heredia; 2009.

24. Rey de Castro J, Rosales-Mayor E. Cansancio y somnolencia durante el desempeño laboral de los conductores interprovinciales: experiencia peruana y planteamiento de propuestas. Rev Peru Med Exp Salud Publica. 2010; 27(2): 237-42.

25. George CF, Nickerson PW, Hanly PJ, Millar TW, Kryger MH. Sleep apnea patients have more automobile accidents. Lancet. 1987;2(8556):447.

26. Teran-Santos J, Jimenez-Gomez A, Cordero-Guevara J. The association between sleep apnea and the risk of traffic accidents. Cooperative Group Burgos-Santander. N Engl J Med. 1999; 340(11): 847-51.

27. Ellen RL, Marshall SC, Palayew M, Molnar FJ, Wilson KG, Man-Son-Hing M. Systematic review of motor vehicle crash risk in persons with sleep apnea. J Clin Sleep Med. 2006;2(2):193-200.

28. Rey de Castro J, Alvarez J, Gaffo A. Síntomas relacionados a trastornos del sueño en supuestos sanos que asisten a un centro de atención primaria de salud. Rev Med Hered. 2005;16(1):31-8.

29. Perú, Ministerio de Transportes y Comunicaciones. Modificatoria del reglamento nacional de licencias de conducir vehículos automotores y no motorizados de transporte terrestre. Decreto Supremo N ${ }^{\circ}$ 001-2009 de 09 de enero de 2009. Lima: MTC; 2009. 
30. Rey de Castro J, Tagle I, Escalante N. Síndrome apneahipopnea obstructiva del sueño (SAHOS): Propuesta para su diagnóstico y tratamiento. Comité Apnea Sueño de la Sociedad Peruana de Neumología. Rev Soc Peru Neumología. 2001; 44(1): 24-48.

31. Durán J, Rey de Castro J. Diagnóstico domiciliario del síndrome de apneas-hipopneas del sueño. Pulmón. 2002; 2(2): 3-10.

32. Durán J,Rey de CastroJ, De la Torre G,Aguirregomoscorta J. Síndrome de apneas-hipopneas durante el sueño. En: Villasante C, editor. Enfermedades respiratorias. Madrid: Aula Médica Ediciones; 2002. p. 265-81.

33. Nunez R, Rey de Castro J, Socarras E, Calleja JM, Rubio R, Aizpuru F, et al. Validation study of a polygraphic screening device (BREAS SC20) in the diagnosis of sleep apnea-hypopnea syndrome. Arch Bronconeumol. 2003; 39(12): 537-43.

34. Rey de Castro J, Ferreyra J, Rosales E. Método simplificado para el diagnóstico del síndrome de apneahipopneas del sueño (SAHS). A propósito de una serie de casos empleando el polígrafo respiratorio BREAS SC-20. Rev Med Hered. 2007; 18(2): 59-67.

35. Ferber R, Millman R, Coppola M, Fleetham J, Murray $\mathbf{C F}$, lber $\mathbf{C}$, et al. Portable recording in the assessment of obstructive sleep apnea. ASDA standards of practice. Sleep. 1994; 17(4): 378-92.

36. Rechtschaffen A, Kales AA. A manual of standardized terminology techniques and scoring system for sleep stages of human subjects. Bethesda: U. S. National Institute of Neurological Diseases and Blindness, Neurological Information Network; 1968.

37. Iber C, Ancoli-Israel S, Chesson AL, Quan SF. The New Sleep Scoring Manual -The Evidence Behind The Rules. J Clin Sleep Med. 2007;3(2):107.

38. [No authors listed]. EEG arousals: scoring rules and examples: a preliminary report from the Sleep Disorders Atlas Task Force of the American Sleep Disorders Association. Sleep. 1992; 15(2): 173-84.

39. Douglas NJ, Thomas S, Jan MA. Clinical value of polysomnography. Lancet. 1992; 339(8789): 347-50.

40. Duran Cantolla J, Esnaola Sukia S, Rubio Aramendi R, Egea Santaolalla C. Validity of a portable recording system (MESAM IV) for the diagnosis of sleep apnea syndrome. Arch Bronconeumol. 1994; 30(7): 331-38.

41. Lloberes P, Montserrat JM, Ascaso A, Parra O, Granados A, Alonso $\mathbf{P}$, et al. Comparison of partially attended night time respiratory recordings and full polysomnography in patients with suspected sleep apnoea/hypopnoea syndrome. Thorax. 1996; 51(10): 1043-47.

42. Ballester E, Solans M, Vila X, Hernandez L, Quinto L, Bolivar I, et al. Evaluation of a portable respiratory recording device for detecting apnoeas and hypopnoeas in subjects from a general population. Eur Respir J. 2000; 16(1): 123-27.
43. Mallampati SR, Gatt SP, Gugino LD, Desai SP, Waraksa B, Freiberger D, et al. A clinical sign to predict difficult tracheal intubation: a prospective study. Can Anaesth Soc J. 1985; 32(4): 429-34.

44. Nuckton TJ, Glidden DV, Browner WS, Claman DM. Physical examination: Mallampati score as an independent predictor of obstructive sleep apnea. Sleep. 2006; 29(7): 903-8.

45. Rosales E. Estudio de validez y confiabilidad de la escala de somnolencia de Epworth en población peruana y modificación de la escala para población que no conduce vehículos motorizados [Tesis de Maestría]. Lima: Facultad de Medicina, Universidad Peruana Cayetano Heredia; 2009.

46. Chobanian AV, Bakris GL, Black HR, Cushman WC, Green LA, Izzo JL Jr, et al. Seventh report of the Joint National Committee on 259, Detection, Evaluation, and Treatment of High Blood Pressure. Hypertension. 2003; 42(6): 1206-52.

47. Barbe F, Sunyer J, de la Pena A, Pericas J, Mayoralas LR, Anto JM, et al. Effect of continuous positive airway pressure on the risk of road accidents in sleep apnea patients. Respiration. 2007; 74(1): 44-49.

48. George CF. Reduction in motor vehicle collisions following treatment of sleep apnoea with nasal CPAP. Thorax. 2001; 56(7): 508-12.

49. Krieger J, Meslier N, Lebrun T, Levy P, Phillip-Joet F, Sailly JC, et al. Accidents in obstructive sleep apnea patients treated with nasal continuous positive airway pressure: a prospective study. The Working Group ANTADIR, Paris and CRESGE, Lille, France. Association Nationale de Traitement a Domicile des Insuffisants Respiratoires. Chest. 1997; 112(6): 1561-66.

50. National Health and Medical Research Council. Effectiveness of nasal continuous positive airway pressure (nCPAP) in obstructive sleep apnea in adults. Camberra: NHMRC; 2000.

51. Grupo Español de Sueño. Consenso nacional sobre síndrome de apneas-hipopneas del sueño. Arch Bronconeumol. 2005; 41(Supl 4): 1-110.

52. Morgenthaler TI, Kapen S, Lee-Chiong T, Alessi C, Boehlecke B, Brown T, et al. Practice parameters for the medical therapy of obstructive sleep apnea. Sleep. 2006; 29(8): 1031-35.

53. Tregear S, Reston J, Schoelles K, Phillips B. Obstructive sleep apnea and risk of motor vehicle crash: systematic review and meta-analysis. J Clin Sleep Med. 2009; 5(6): 573-81.

54. De Mello MT, Bittencourt LR, Cunha Rde C, Esteves AM, Tufik S. Sleep and transit in Brazil: new legislation. J Clin Sleep Med. 2009; 5(2): 164-66.

Correspondencia: Jorge Rey de Castro Mujica

Correo electrónico: jorgerey@rcp.net.pe 